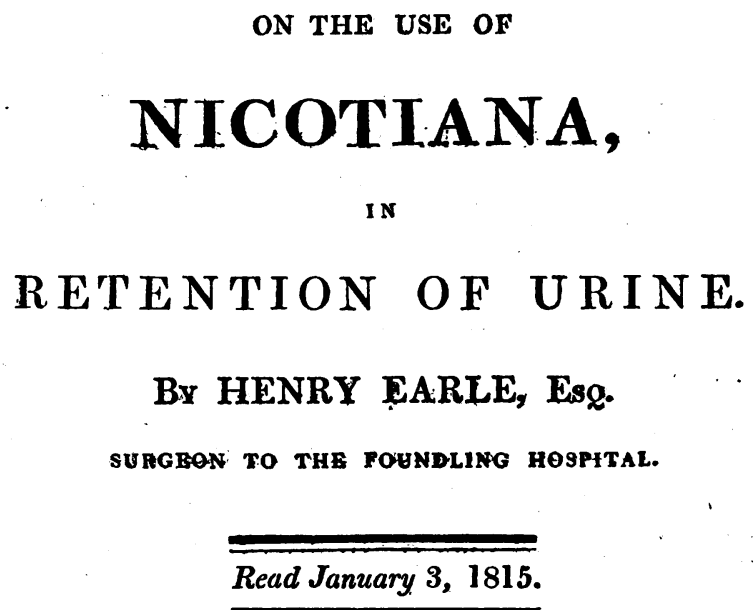

IHE foltowing observations on an efficacious mode of relieving some of the most alarming cases of retention of urine, will not, I trust, be devoid of interest to the Society, when the frequency of the complaint, and the difficulties which occasionally accur in the treatment of it are considered.

The facts in illustration of the practice are not numerous, but sufficient to warrant my laying the subject before the public, to be confirmed or confuted by subsequent experience.

The causes of retention of urine are so various, that it is not my intention to enter at large into the subject; more especially as the plan of treatment which I shall speak of, is only applicable to 
particular cases: I shall therefore confine myself to a brief consideration of the nature of such cases.

It is well known, that persons who have been long subject to strictures in the urethra, but who are still able to void their urine in a small stream, are liable, from accidental causes, to have a complete retention, and are incapable of expelling the contents of their bladder. This arises in some cases from the calibre of the urethra being still further diminished by attacks of inflammation, but more frequently from the spasmodic state of the muscles of the urethra.

The same effect may be produced in persons labouring under stricture, by retaining their urine beyond the usual period for expulsion. Even in a state of perfect health, if we suffer the bladder to be over distended, whereby the muscular fibres are stretched beyond their natural sphere of contraction, every one has experienced that a greater effort is required, and the aid of the abdominal muscles is obliged to be called in, to overcome the resistance afforded by the neck of the bladder. This, I conceive, arises from the disturbance of that nice equilibrium which naturally exists between the expelling and resisting power. When disease has existed for some time in the urethra, and a permanent obstacle is afforded to the egress of the urine, the expelling power is exerted with greater force : the bladder consequently becomes 
thicker, more muscular, and contracts on a smaller quantity of fluid.

It happens not unfrequently, that the permanent stricture may be of such a nature as not to admit of the introduction of any instrument into the bladder, even under the most favourable circumstances. I need scarcely add, that a spasmodic state of the urethra would not facilitate such attempts. Other cases again occur, in which perhaps an instrument can be passed, when the urethra is in a more tranquil state, but where it would be highly injudicious, and often impracticable to introduce such instruments under circumstances of irritation, by which attempts the spasm would be increased, and the patient rendered liable to returns of retention, were we to succeed in the first instance.

In all such cases it is highly desirable to overcome the retention by other means than the introduction of instruments. For this purpose purgatives, general and local bleeding, warm baths, and tinctura ferri muriatis are commonly resorted to. With respect to purgatives, their action necessarily requires more time, than, from the urgency of the symptoms, is frequently admissible. The other remedies are highly useful, and will frequently fulfil every indication; occasionally, however, they are unavailing, and we are compelled to resort to operations for relieving the distended bladder. 
In offering another powerful auxiliary to be adopted in cases which have resisted the ordinary means employed, I hope to confer some benefit on society. The medicine to which I allude is the Nicotiana, to be exhibited as an enema, in the form either of smoke or of infusion.

The powerful effect of this medicine in strangulated hernia, first led me to propose its administration in obstinate cases of retention of urine, in a paper on diseases of the urethra, which I drew up some years since for a Medical Society. I shall now proceed to relate the cases in which $I$ have had an opportunity of ascertaining its effects.

In October, 1812, I was requested to attend Charles Wright, for a retention of urine, of which he gave the following history. When about eigh teen years of age, he had suffered severely from gonorrhœa and hernia humoralis; from this period he dated the complaint in his urethra. He was now thirty-five : during this time he had been in a gentleman's service, as groom, and had been obliged to ride a great deal. The stream of water gradually diminished in size, accompanied with frequent and urgent calls, until about two years before the present period, when, from being obliged to remain a long time on horseback, he had a retention of urine, accompanied with so much inflammation, that an abscess formed in the perineum, which burst and became fistulous. For this 
complaint he had been for some time under a surgeon's care, who attempted to pass bougies, but never succeeded in reaching the bladder. He had latterly been in the habit of passing a metallic bougie for himself, which was the probable cause of the present retention and inflammation.

On examination, I found a firm obscurely elastic tumour, about the size of a pigeon's egg, situated immediately on the urethra, at the lower part of the scrotum. This was about the point to which he had been accustomed to pass the instrument. The surrounding scrotum was healthy, which led me to refer the present abscess rather to the irritation of the bougie, than to any effusion of urine, which generally diffuses itself more extensively. The abscess had been about three days in forming, accompanied by great pain and fever, and he had not been able to void his urine for the last eighteen hours. I immediately made a free incision into the abscess, and let out about 3iv. of very fetid pus. I directed him to sit in warm water, and ordered a common clyster to be thrown up. As he was still unable to make water after the trial of these means, I desired him to take fifteen drops of tinctura ferri muriatis every ten minutes, in barley water. He continued it for nearly three hours; the medicine produced nausea and headache, but still no water passed. I now attempted to introduce a bougie, but could not get beyond six inches; the introduction thus far was productive 
of great pain. His symptoms were now very urgent, for although the bladder was not greatly distended, yet from the long existence of disease it had probably become much thickened, and was. very irritable. Apparently no alternative now remained but an operation; and as the bladder could not be satisfactorily felt above the pubes, and the perineum was much thickened and diseased, I determined in my own mind to puncture from the rectum. Previous, however, to resorting to this. ultimum remedium, I was desirous of trying the effect of the Nicotiana.

With this view I procured some common tobacco, and not having any scales was obliged to guess at about two drachms, on which I poured a pint of boiling water. Eight ounces of the infusion were thrown up, and with some difficulty retained. After about ten minutes the patient became very faint and sick; a clammy sweat broke out over his whole body, his pulsa.became feeble and intermittent, and the urine began to dribble away to a considerable amount. 'The contents of the rectum were now suffered to come away, consisting of the infusion mixed with fæculent matter. As he still continued very faint, a small quantity of brandy was given, which quickly restored him. The effect of the Nicotiana in this instance was most decidedly beneficial, though the symptoms produced were certainly alarming, arising probably from the uncertain strength of the infusion. 
I continued to attend the patient for some time; the abscess in the scrotum was unconnected with the canal of the urethra, and healed without difficulty. After some days I commenced passing bougies; at first I could not get beyond six inches, but by - a few applications of the caustic, I succeeded in passing a tolerable sized one about eight inches. The fistulous opening in the perineum I laid open, and by a compress and sticking-plaster, effectually prevented the further escape of urine. He was in every respect much relieved, and there was a good prospect of his ultimate recovery, when he was obliged to leave London, and I have never since heard of him.

The next case in which I tried tobacco, occurred about three months after, in a gentleman who had been for many years subject to strictures, and for want of proper medical assistance, had suffered the disease to proceed to a most alarming extent. When he first applied to me, he declared that he had not, for a long time, been able to sleep for the space of one hour, and he was frequently obliged to make water every quarter of an hour. His general health was much impaired by want of rest, and the continual irritation under which he laboured. Generally the water came away in a fine hair stream, but at times it passed grittatim. On examining his urethra, I found a stricture about four inches down, which I passed with a small bougie, but could not succeed with the finest 
in getting beyond six inches. After several inef: fectual trials with common and catgrat bougies, it became necessary to resort to the caustic. After six applications he was attacked with a retention of urine, accompanied with great pain and anxiety. All the common remedies were in turn resorted to: he was bled, clystered, placed in a warm bath, and took the tinctura ferri muriatis, but all failed in producing the desired effect. The success I had met with in the last mentioned case led me to resort again to the Nicotiana; an infusion of the strength of one drachm to eight ounces was made and thrown up. In about a quarter of an hour he became rather faint and complained of languor, and in a few minutes more the water began to flow from him. The effect of the medicine in this case was by no means so violent as in the former, but equally efficacious.

As I had evidently gained ground with the argentum nitratum, I again resorted to it, and after applying it thirty-five times, by cautious perseverance I succeeded in reaching the bladder, and freed my patient from all his sufferings. During this period he was at two several times again attacked with retention, and was relieved by the tobacco infusion, to which I at once resorted, without subjecting him to the delay of other medicines.

The third case occurred in the course of last summer: I was sent for to attend a young man 
labouring under retention of urine. I found that he had been for years subject to strictures, but had always been able to void his urine until that morning, when, on rising, he was unable to discharge the contents of his bladder. He had been in the habit of passing a small whalebone bougie, which he now attempted in vain to introduce. He called on a neighbouring apothecary, who immediately attempted to pass a catheter, and used great force, which was followed by a copious flow of blood, but no urine. He was next bled from the arm, and some opening medicine was administered, and as he was still unable to make water, I was called in.

I saw him about three o'clock in the day, the retention having existed from the preceding night. He was still bleeding freely from the urethra, and had a most urgent desire to makc water. I directed him to take the tinctura ferri muriatis, and to sit in a tub of warm water. He took 18 doses of the tincture without any perceptible effect but nausea. I now attempted to pass a bougie, but when about eight inches down it quitted the right tract and was readily detected, by introducing the finger per anum, passing between the bladder and the rectum. I immediately withdrew the bougie, which had caused much pain, though introduced with the utmost care and gentleness, and ordered an infusion of Nicotiana, of the strongth of one drachm to eight ounces, to be used as an enema. 
I was under the necessity of leaving him, to visit a patient a short distance from town, but on my return, in less than two hours, I was informed that a short time after the injection he had been very faint, and had perspired copiously, during which time the urine flowed from him in a stream.

I directed him to keep quiet, and whenever he made water to press with his hand on the perineum, to prevent, if possible, the escape of any urine by the false passage: he had no return of the retention, and by the above precaution had no effusion of urine. It is most probable that in this case the retention was kept up, and symptoms aggravated by the injudicious introduction of the catheter in the first instance, without resorting to any other means. As the urethra had been pervious to the passage of urine the preceding night, it was evident that the complaint depended on spasm, which might have been relieved by warm bathing, or the cautious introduction of a bougie. Should such a case occur to me in practice, I should be induced to try the injection of warm olive oil into the urethra, as I understand that this plan has been very successfully adopted in Italy, in cases of retention arising from spasm combined with permanent stricture.

These are the only instances in which $I$ have had an opportunity of trying the effect of the Nicotiana in retention of urine. $I$ have ventured to 
detail the cases, as illustrative of three different causes of retention, each, however, corresponding in the impracticability of the introduction of in:struments, and each having resisted the ordinary modes of relief.

The operation of Nicotiana varies much in different individuals, and is influenced in some degree by the habit of smoking or chewing tobacco. It generally acts very powerfully, and I have known it produce most alarming syncope; it ought not, therefore, to be adopted indiscriminately in slight cases, but reserved for instances where more simple means have failed. It is, probably, in consequence of the occasional violence of its action that medical men have been deterred from using it, except in cases of strangulated hernia, as I am not aware of its having been before tried in retention of urine.

Its virtues as an antispasmodic are so eminent, that $I$ was induced to try it once in a very bad case of tetanus, in which, although it afforded a temporary alleviation from spasm, the exhibition of the enema caused so much agitation that it was not persevered in. Should such a case occur again, or should I happen to meet with a case of hydrophobia, I should be much inclined to try the effect of an extract of Nicotiana made into a suppository and placed up the rectum. This form of administering the medicine would embrace many important advantages. Its strength might be bet- 
ter regulated than by an infusion or the smoke. It would produce little or no irritation; it might be easily removed if found to operate too violently; and, lastly, if its effect were salutary, it might be retained for a much greater length of time than the injection. As the case to which I have just alluded was attended with some peculiarities, the particulars of it may not be unacceptable to the Society, though unconnected with the present subject.

\section{Case of Tetanus.}

Joseph Owen lacerated his great toe with a block of wood. There was nothing remarkable in the appearance of the wound, which was granulating, when on Tuesday, Febt. 26, about three weeks from the receipt of the accident, he was seized with pain and stiffness in the muscles of his back, and was troubled with severe cramps in his legs and thighs. These symptoms rapidly increased in violence, and in a short time his jaw became affected; the muscles of the abdomen and neck afterwards partook in the affection. In a word, all the voluntary muscles were affected with tetanus. I found him in this state with a countenance expressive of the greatest terror and anxiety; his pulse was strong and full, beating about 140 in a minute, and his whole body was covered with a profuse sweat. I immediately bled him to the amount of twenty ounces, and gave him pulveris 
ipecacuanhæ comp. gr. xv., calomelanos gr.v. The spasms were much relieved by the loss of blood, were less frequent, and of shorter duration. A physician who now saw him, ordered him to take large quantities of wine and sago, and to continue taking the pulv. ipec. comp. gr. vo omni bihorâ. The wine aggravated the complaint so much, that its use was soon discontinued. I now tried the enema of tobacco smoke, the result of which has been related above. Towards night the spasms were again very violent, and ten ounces more of blood were taken away, after which he appeared much easier; his pulse became softer, and he broke out into a profuse sweat; the muscles of the abdomen relaxed, and were evidently affected by respiration. The blood which had been drawn in the morning was mostaremarkably buffed and cupped. The following morning I found him much better, and he had slept for some hours in the night. He had not passed any fæces, and felt very desirous of easing, himself, but was prevented by the spasmodic affection of the muscles whenever he exerted himself in the slightest degree. I directed him to have clysters with oleum ricini thrown up, which returned without any fæculent matter. He experienced much difficulty, and an aggravation of the spasms whenever he attempted to make water, which was small in quantity and very turbid. He was so much worse towards evening, that twenty ounces more blood were taken from his arm, which again procured him ease 
and some sleep. In the course of the night he passed a fluid stool; the following morning, his pulse being still full and hard; and the spasms being occasionally violent, though much less frequent, I took twelve ounces more from him. He experienced so much ease after each bleeding, that towards evening he requested to be again bled, being persuaded that it was the only means of obtaining a quiet night; accordingly twelve more ounces were taken. He passed a tranquil night, and slept three hours at one time; early in the morning he had three copious evacuations. He was now considerably better in every respect; his pulse was much softer, and he was able to take some nourishment; his countenance was less anxious, and he no longer talked of dying. During the day he was much agitated by his relations coming to visit him, and at night it was necessary to bleed him to the amount of sixteen ounces. On Saturday morning he was much better; he had slept a great deal during the night, and was much refreshed. He said that he felt much improved, but very weak. Some arrow-root and jelly with a little wine were given to him ; but the wine reproduced the spasms, so as to render it necessary again to have recourse to the lancet at night. All Sunday he was so much better that I entertained confident hopes of his surviving; the principal pain he complained of was across his back and belly, and in one thigh. On Monday he continued to improve, but unfortunately his friends paid him 
another visit, and at his request gave him some porter, which reproduced the spasms, but in a much diminished degree. Towards evening he complained of weakness, his countenance had changed much during the day, his pulse became very frequent, and when sleeping he rambled a little. During the night he sunk rapidly, and the following morning expired. The blood, to the very last, exhibited the strongest marks of inflammation.

\section{Dissection.}

Nothing particular was found in the head; the veins of the pia mater were rather turgid with blood. The thoracic and abdominal viscera were healthy. On the surface of each psoas magnus muscle there was an effusion of blood, on removing which the muscular fibres were seen much lacerated and altered in texture, being quite soft and giving way under the fingers. This lacerated appearance occupied nearly the whole thickness of the muscles to some extent. A similar effusion of blood had taken place in the sheath of the right rectus abdominis, and the muscular fibres were torn and quite soft, like the muscles of an animal that had been hunted to death.

Though this case terminated fatally, I conceive that the appearance of the blood and the remission of pain on bleeding, fully warranted my pro- 
secuting this plan. The muscular fibres being actually torn asunder, sufficiently prove the violence of the attack; and I think that although I was not so fortunate as to preserve his life, I rendered his severe sufferings morte supportable. Blood-letting in tetanus has been much censured by different authors, but I must hesitate in subscribing to their opinions, which I conceive have been formed without sufficient trial, or rather, which have been adopted from one generation to another without the acquisition of any new facts. I am induced to form this conclusion from meeting with the following passage in Cullen. He says, "B Blood has often been drawn in this disease, but it never exhibits any inflammatory crust, and all accounts seem to agree that the blood drawn seems of a looser texture than ordinary, and that it does not coagulate in the usual manner."

Now either the case which I have just related was anomalous, or Cullen has adopted opinions without sufficient grounds. As I do not possess any other facts on this head, I cannot pretend to decide on the subject, but scruple not to declare that should such a case again occur, with as much arterial action, and with such appearances of the blood, I should certainly adopt a similar line of practice, combined with the use of the Nicotiana. 